\title{
Desafíos hacia una evaluación sistémica de la educación terciaria en Uruguay
}

\author{
Challenges towards a systemic evaluation of tertiary \\ education in Uruguay
}

Desafios para uma avaliação sistêmica do ensino superior no Uruguai

ISSN 1688-9304 - DOI: 10.18861/cied.2020.11.2.2991

Emiliano Clavijo*1

https://orcid.org/0000-0003-0670-4669

Agustina Marques**2

https://orcid.org/0000-0002-7373-6567

Cecilia Rodríguez ***3

https://orcid.org/0000-0003-3236-344X

Fecha de recibido: 23/05/2019

Fecha de aprobado: 06/03/2020

\section{Resumen}

La Ley General de Educación N 18.437 (LGE) aprobada en 2008 impulsó cambios institucionales sustantivos en el contexto de la educación terciaria (ET). En el ámbito de la evaluación surgen novedades tales como la creación del Instituto Nacional de Evaluación Educativa (INEEd) o la reciente creación del Instituto Nacional de Acreditación y Evaluación de la Educación Terciaria (INAEET). Vistos estos antecedentes, la discusión de dimensiones e indicadores para la evaluación de la ET desde un enfoque sistémico resulta más que pertinente a una década del inicio de este proceso. En este sentido, a partir de una revisión bibliográfica sobre las temáticas en el campo de la ET se proponen siete dimensiones para abordar la evaluación en ET desde una perspectiva sistémica. Las mismas incluyen: los diseños institucionales, el factor territorial -que cobra mayor relevancia en el marco del proceso de descentralización que experimentó la política educativa a nivel superior-, aspectos sociodemográficos, características del proceso educativo, asuntos vinculados a la currícula, asuntos vinculados a la dimensión presupuestal y el creciente proceso de internacionalización. Mediante el análisis documental de informes institucionales y leyes, entre otros, se describen estas dimensiones para el caso uruguayo. Dado que el proceso de diversificación institucional experimentado en el ámbito de la ET implicó el desarrollo de estrategias particulares por parte de las instituciones, consideramos que el principal desafío radica en lograr consenso entre las instituciones del sistema para poder avanzar en la evaluación de las dimensiones consideradas.

Palabras clave: evaluación; educación terciaria; sistema educativo; política educativa; Uruguay.

\footnotetext{
Abstract

The General Education Law ( $\mathrm{N}^{\circ}$ 18.437) passed in 2008 drove important institutional changes in the sphere of Higher Education (HE). New projects emerged regarding the area of evaluation, such as the creation of the National Institute of Educational Evaluation, or the recent creation of the National Institute of Accreditation and Evaluation of Tertiary
} 
Education. Given these precedents, the discussion about dimensions and indicators for the evaluation of $\mathrm{HE}$ from a systemic viewpoint is more than relevant, having elapsed a decade since the onset of this process. In this sense, departing from a literature review on topics related to $\mathrm{HE}$, we propose seven dimensions to address an evaluation of $\mathrm{HE}$ from a systemic perspective. These include: institutional designs, territorial issues that are gaining more attention due to the decentralization process in the higher education policy, socio-demographic aspects, characteristics of the educational process, curriculum issues, budgetary structure, and a growing process of internationalization. Through examination of institutional reports and laws, among others, we describe these seven dimensions for the Uruguayan case. Since the process of institutional diversification experienced in $\mathrm{HE}$ implied the development of particular strategies by the different institutions, we consider that the biggest challenge relies in achieving consensus between the system institutions in order to move up towards the evaluation of the above mentioned dimensions.

Keywords: evaluation; tertiary education; education system; educational policy; Uruguay.

\section{Resumo}

A Lei Geral de Educação $n^{\circ} 18.437$ (LGE), aprovada em 2008, levou a mudanças institucionais substanciais no contexto do ensino superior (ES). No campo da avaliação, surgem desenvolvimentos como a criação do Instituto Nacional de Avaliação Educacional (INEEd) ou a recente criação do Instituto Nacional de Acreditação e Avaliação do Ensino Superior (INAEET). Em face desse cenário, decorrida uma década do início deste processo, a discussão de dimensões e indicadores para a avaliação do ES a partir de uma abordagem sistêmica é mais do que pertinente. Nesse sentido, com base em uma revisão bibliográfica sobre os tópicos no campo da ES, sete dimensões são propostas para abordar a avaliação na ES de uma perspectiva sistêmica. Estes incluem: desenhos institucionais, o fator territorial que se torna mais relevante no marco do processo de descentralização experimentado pela política educacional em um nível superior, aspectos do processo sociodemográfico e educacional, questões relacionadas ao currículo, dimensão orçamentária e o crescente processo de internacionalização. Através da análise documental de relatórios e leis institucionais, entre outras, essas dimensões são descritas para o caso uruguaio. Embora o processo de diversificação institucional experimentado no campo da ES abranja o desenvolvimento de estratégias específicas pelas instituições, acreditamos que o principal desafio está em obter consenso entre as instituições do sistema para poder avançar na avaliação das dimensões consideradas.

Palavras-chave: Avaliação; Ensino superior; Sistema educativo; Política educativa; Uruguai

\section{Planteamiento del problema: pensar la evaluación en ET en clave sistémica}

La Educación Terciaria (ET) en Uruguay abarca tanto al conjunto de "universidad(es) pública(s) y universidades e institutos universitarios privados", es decir instituciones de educación superior, como a un grupo "heterogéneo de ofertas de educación terciaria no universitaria constituido por institutos de formación docente, cursos técnicos dependientes del Consejo de Educación Técnico-Profesional y escuelas y centros dependientes de ministerios" (Contera, 2008: 533). También podrían incluirse en esta categorización las ofertas educativas que brindan las instituciones de formación policial y militar (formación de oficiales y licenciaturas). Se trata de un subsector de políticas que ha experimentado una serie de transformaciones a lo largo de una década, fundamentalmente a raíz de la aprobación de la Ley General de Educación (Parlamento, 2008). 
Actualmente se vislumbran nuevas interrogantes respecto al sistema de ET. Por un lado las incertidumbres propias que traen consigo los ciclos electorales y, por otro, las restricciones presupuestarias. A su vez la ET se halla en un contexto de transición entre definiciones políticas y expectativas intrínsecas a su ámbito.

Recientemente, el máximo jerarca de la Universidad de la República (UdelaR) Rodrigo Arim, al ser interrogado acerca de la colaboración de la institución con el resto de los actores del sistema público y en particular con el sector de educación secundaria declaró que "falta coordinación efectiva. [...] Es responsabilidad de todos mejorar el diálogo con [el Consejo de Educación] Secundaria con respecto a las transiciones, tener hasta estadísticas comunes" (Radio Uruguay, 29 de enero de 2019)i. Tales declaraciones brindadas por el Rector de la UdelaR plantean una voluntad colaborativa con otros actores del sistema. Entonces, ¿por qué no pensar en un sentido amplio y extender esta propuesta a todas las instituciones del sistema educativo?

El objetivo de este trabajo es realizar un abordaje reflexivo de dimensiones que brinden insumos para la discusión de un proceso de evaluación de la educación terciaria en Uruguay desde una perspectiva sistémica. Esto es de particular relevancia en un contexto de diversificación institucional, proceso que responde a diversos factores tales como el crecimiento de la oferta académica y el desarrollo de carreras técnicas y tecnológicas. Asimismo se constatan cambios institucionales a través de ajustes o innovaciones en el ámbito de la ET pública en Uruguay (Bentancur y Clavijo, 2016), como una nueva universidad pública (Universidad Tecnológica). También se percibe un progresivo avance y consolidación de ofertas académicas en universidades e instituciones universitarias privadas.

Considerando este escenario surge la inquietud acerca del alcance e intensidad del diálogo interinstitucional, con énfasis en aspectos que atañen a la evaluación desde una visión sistémica. Ello no implica reflexionar acerca de estos asuntos desde una óptica comparativa entre las instituciones. Por el contrario, se trata de dar luz a las particularidades y generalidades que presentan los actores del sistema con la finalidad de pensar en dimensiones e indicadores para la evaluación en ET. Ciertamente, estos asuntos presentan condicionamientos tales como la posibilidad de construir el dato, el contexto de su elaboración y procesamiento, además de los énfasis y las necesidades que se presentan institucionalmente.

En consecuencia se plantea la siguiente pregunta de investigación: ¿Cómo abordar la evaluación de las políticas educativas de ET en Uruguay desde un enfoque sistémico? Para acercarnos a una respuesta se avanzó en la formulación de siete dimensiones tomando como insumo antecedentes bibliográficos de cada una de las áreas de interés abordadas. Esto implica reflexionar sobre los condicionamientos y habilitaciones que presentan los factores de naturaleza institucional, territorial, sociodemográfica, educativa, curricular y presupuestal, además de la internacionalización como un proceso creciente en el contexto de la ET.

Fundamentación teórica: dimensiones pertinentes para la evaluación

A continuación se abordan la identificación y descripción de tópicos que han sido objeto de investigación en evaluación educativa, que orientan la discusión de dimensiones de pertinencia y plantean el desafío de asumir una concepción sistémica. Principalmente se apuntó a la selección de diversos ejes que han formado parte de la agenda educativa ya sea por factores domésticos ligados a las reformas en el sector o bien factores exógenos 
que responden a la agenda internacional en este ámbito. Ciertamente pueden integrarse otros elementos a la discusión por lo que no se trata de un asunto que se saldará en estas líneas. Por el contrario, se pretende brindar un insumo con la finalidad de estimular la discusión acerca del diseño e implementación de políticas educativas en clave sistémica.

\section{Diseño Institucional}

Algunos ejes clave para orientar el análisis del diseño institucional refieren a aspectos estructurales y dinámicos con atención a tópicos de carácter institucional en términos de las reglas de juego, el abordaje de los paradigmas de ideas en torno a las visiones de política educativa y el enfoque de actores que interactúan en un contexto institucional (Bentancur, 2012). Ello implica posicionarse desde una visión pluralista de la política, en la que los sujetos involucrados manifiestan definiciones sobre la problemática a abordar así como también sus objetivos y costos a afrontar (Lindblom, 1992).

En cuanto a la primera dimensión podría ser relevante identificar aspectos organizativos, propios de las estructuras que delinea el marco legal (Constitución, leyes, decretos, etc.), persiguiendo la tradición del institucionalismo normativo (March y Olsen, 1989) o el institucionalismo histórico (Pierson y Skocpol, 2008). Ello podría incluir aspectos vinculados a estructuras de gobierno o a los organigramas que presenta cada institución. En ello puede indagarse por instancias de rendición de cuentas de carácter vertical, horizontal y/o societal, reglas de juego para el desempeño de la docencia, entre otras posibles.

Un segundo factor es el plano de las ideas que consiste en visualizar los diseños institucionales como resultado de ideas de política o ideas programáticas de gobierno y finalmente y en términos filosóficos, orientados a dimensiones ideológicas (Schmidt, 2008). En esta dimensión cobra fuerza el tipo de provisión del servicio (público/privado), las ideas del elenco gubernamental en términos sistémicos, es decir, las propuestas de los partidos políticos frente a la temática, o también aspectos filosóficos, es decir, aportes ideológicos que sustentan modelos de diseño institucional en términos de lo que Hall (1993) denomina paradigma de ideas.

Finalmente, un enfoque de actores implica tomar en consideración el juego político de los actores: identificar roles, recursos y estrategias en los procesos de decisión de políticas (Dente y Subirats, 2014) además de las redes de política que entablan los actores (Marsh y Rhodes, 1992) dentro de las instituciones educativas, entre instituciones y más allá de las instituciones del sistema: actores políticos partidarios, empresas, sociedad civil, etc.

\section{Territorialización}

El análisis de los procesos de descentralización territorial educativa abordada desde la educación primaria y media ha considerado distintos niveles de poder político, lo que sugiere diversos modelos de descentralización y la necesidad de su evaluación (Di Gropello, 2004). Se expresa que la descentralización territorial educativa permite prestar un servicio educativo que se condiga con la heterogeneidad territorial pero cuyos resultados deben presentar una igual calidad (Calero, 2006).

La ET en el mundo ha avanzado desde la concentración en universidades tradicionales a su regionalización y departamentalización. Para el caso latinoamericano es especialmente significativo dada la tendencia centralizante en la toma de decisiones políticas de las grandes ciudades hacia el resto del territorio del país de pertenencia, 
tratándose mayoritariamente de ciudades capitales. Esta tendencia se caracteriza por la concentración de las actividades económicas. Esto presenta, además, implicancias de carácter sociocultural que pautan un claro clivaje entre lo que ocurre en estas áreas urbanas frente al resto del territorio. La descentralización territorial surge en respuesta a un problema de inequidad en el acceso a la ET en función del origen geográfico (Rangel, 2011).

Tanto la descentralización como la regionalización deben analizarse desde el punto de vista de sus implicancias, alcances y resultados de política. La nueva oleada de descentralización universitaria en las universidades latinoamericanas, sumado a los cambios en las tecnologías de la comunicación y en los medios de transporte, trajo consigo nuevos debates respecto al sistema educativo y la sociedad (Rama, 2015). Por esto parece relevante generar indicadores educativos que puedan dar cuenta a su vez del proceso de descentralización y de regionalización en el sistema de ET. En consecuencia, el territorio no actúa como una mera variable contextual sino que atraviesa aspectos de carácter social, económico, político y cultural.

\section{Aspectos sociodemográficos}

La ET se ha expandido en las últimas décadas a lo largo del mundo (Archer, 2005; Shavit, Arum y Gamoran, 2007; Chowdry, Crawford, Dearden, Goodman y Vignoles, 2008). Esta expansión ha vuelto a posicionar el estudio de la relación de la ET con las clases sociales. Por ejemplo, algunos autores señalan que la influencia del hogar de origen disminuye en las sucesivas transiciones educativas mientras que otros sostienen que esta influencia se potencia significativamente en la transición a la ET (Shavit, Arum y Gamoran, 2007; Chowdry, Crawford, Dearden, Goodman y Vignoles, 2008).

A pesar de la expansión en la oferta algunos sujetos no participan en educación postsecundaria, siendo uno de los factores explicativos la inequidad en el acceso a la ET de acuerdo a criterios de estratificación social. Asimismo se sugiere que la expansión de la demanda entre quienes no ingresan al sistema de ET se da principalmente en las carreras terciarias técnicas -de dos y tres años de duración- (Shavit, Arum y Gamoran, 2007). Incluso, los sistemas de ET gratuitos presentan inequidades en el acceso. Cuando generación tras generación las clases más favorecidas son las que hacen uso de un bien público esto implica que se han apropiado de este bien (Archer, 2005).

Es de relevancia evaluar, asimismo, otros atributos sociodemográficos que pueden generar desigualdades. Tal es el caso de las relaciones de género en el sistema de ET. Un ejemplo es la segregación ocupacional por género. Los países con mayor segregación en los sistemas educativos tienen mayor probabilidad de presentar segregación en el mercado de trabajo. Ello muestra cómo distintos cursos tomados por mujeres y varones jóvenes los introduce en trayectorias que culminan en ocupaciones diferenciadas por género (Smyth, 2003). En tanto, la dimensión territorial sugiere diferencias sustanciales entre la capital y el resto del país en cuanto a distribución de edades, fecundidad tanto en calendario como cantidad y en calendario e intensidad del trabajo (Paredes y Varela, 2005; Cabella, Fernández y Prieto, 2015). También la matriz de bienestar se comporta de forma diferenciada territorialmente (Trylesinski, 2006) impactando en las decisiones educativas. 


\section{Aspectos del proceso educativo}

Los desafíos presentes en el proceso educativo en la ET se vinculan con el resultado de este proceso y su intrincado relacionamiento con los elementos del mismo. Los logros educativos de los estudiantes son fruto de un complejo conjunto de factores donde cada actor tiene su responsabilidad en el proceso de hallar los mejores caminos dentro de su ámbito de decisiones, responsabilidad compartida tanto por políticos como por administradores, técnicos, directivos y docentes de las instituciones formadoras así como también por los propios alumnos (Ravela, 2016). La evaluación del proceso debe servir, entonces, para reorientar y planificar la práctica educativa. Conocer lo que ocurre en la institución educativa reorientando cuantas veces sea necesario los procesos durante su desarrollo es una de las funciones más importantes de la evaluación.

Es relevante considerar las dimensiones del proceso educativo, lo que implica constatar la situación inicial de los estudiantes, dar cuenta a su vez del proceso administrativo, organizacional, docente y pedagógico del programa. Los resultados alcanzados con los dispositivos educativos son parte del proceso educativo y por tanto es relevante evaluarlos. Se relacionan con el nivel de preparación alcanzado en términos de las capacidades valoradas socioeconómicamente como requeridas. En este sentido son imprescindibles indicadores que midan las capacidades de los nuevos egresados para el desempeño efectivo de su profesión (Unidad de Sistemas de la Información de la Enseñanza, 2014). Asimismo debemos señalar que la discusión puede darse en torno a la evaluación de competencias generales o específicas de cada profesión (Salazar y Chiang, 2007).

\section{Aspectos curriculares}

Los aspectos del diseño curricular están relacionados con las características de la oferta educativa en sí misma. En el plano más general se encuentra la discusión de un currículum basado en competencias o en contenidos (Centro Interuniversitario de Desarrollo, 2008). Otra discusión transversal al diseño curricular es el referido a las diferencias entre el currículo concebido como planes de estudio, llamado currículum, en contraste con la perspectiva del currículo como conjunto de experiencias, denominado currículum vivido, e incluso aquel denominado currículo oculto (Díaz Barriga, 2005).

Son varias las dimensiones relacionadas con el diseño curricular. Por un lado, lo vinculado a las titulaciones otorgadas entendidas como una certificación que acredita un conjunto de conocimientos y capacidades: la carga horaria, el sistema de créditos, los requisitos de ingreso y el perfil de egreso (Marquina, 2004). A su vez, el diseño curricular implica el cómo se imparte el programa: las modalidades educativas, las orientaciones curriculares, los dispositivos de evaluación y la estructura de los planes de estudios (Esquetini y Rodríguez, 2012).

En definitiva, una evaluación del diseño curricular permite conocer y analizar los sistemas de evaluación de aprendizajes y las definiciones conceptuales que los sustentan, así como identificar innovaciones curriculares y pedagógicas que se presenten (Centro Interuniversitario de Desarrollo, 2014). 


\section{Financiamiento}

La inversión en educación es una de las grandes decisiones que deben tomar los estados y, particularmente, quienes toman las decisiones a nivel gubernamental. La provisión del servicio educativo a nivel superior es un aspecto de esta inversión, que inherentemente moviliza abultados recursos. A su vez, el aumento de la demanda de este tipo de educación sumado a una prospectiva de sostenido crecimiento hace de este un tema para debatir y reflexionar a nivel tanto institucional como político y de la sociedad en general. Tal como señalan Estévez y Pérez (2007) la dimensión de los recursos asociados al proceso educativo comprende recursos financieros, además de físicos y humanos.

La cantidad de recursos financieros destinados a la ET es uno de los elementos centrales de las políticas públicas en este sector, que adopta rasgos particulares en un sistema de naturaleza mixta de provisión del servicio, con diversidad de fuentes y esquemas compartidos (Brunner y Villalobos, 2014). Dentro de los recursos financieros asociados a la ET es necesario poder distinguir entre las fuentes de financiamiento para las diferentes instituciones y su composición de modo de poder evaluar esta dimensión a nivel de sistema, al igual que por las implicancias económicas y políticas que acarrean las diversas formas de financiamiento. Por un lado, el aspecto económico debería poder garantizar sostenibilidad en el tiempo y equidad. Por otro, desde un punto de vista político deberían dilucidarse los intereses en juego relativos a la financiación. Este punto es de especial relevancia en un contexto de democratización en el acceso a la ET.

\section{Internacionalización}

Especialmente en las últimas décadas la internacionalización de la ET ha ganado un lugar importante en la agenda de las instituciones de ET de la mano de la globalización, creciendo en cantidad y complejidad (Altbach y Knight, 2007). Si bien surgió inicialmente en los países más desarrollados, América Latina no escapa hoy a este proceso (Didou, 2017).

Es necesario mencionar que existe cierta confusión en torno al significado de la internacionalización y a sus conceptos adyacentes, tales como la transnacionalidad, la binacionalidad o las nociones de universidad cosmopolita o global (Knight, 2015). Por lo tanto, el concepto de internacionalización de la ET es polisémico y abarca varios procesos que implican, por ejemplo, la cooperación y movilidad entre estudiantes y docentes, una internacionalización de la currícula de los programas y la promoción de instituciones de educación terciaria a nivel internacional (Altbach y Knight, 2007).

Asimismo se identifican aproximaciones institucionales muy diferentes, incluso dentro de universidades pertenecientes al mismo sistema de educación terciaria, que generan perfiles de movilidad y cooperación distintos (Bordean y Borza, 2013).

A su vez se ha planteado el análisis de la relación entre la calidad de la educación terciaria y el proceso de internacionalización, que es compleja debido a las múltiples manifestaciones de la internacionalización, además de la dificultad, ya conocida, de definir una educación de calidad. En todo caso, conviven al menos dos enfoques que vinculan calidad e internacionalización. El primero implica analizar en qué medida los procesos de internacionalización en sus diferentes expresiones contribuyen al cumplimiento de los objetivos y fortalecen los modelos educativos. El segundo se relaciona con el conocimiento de la calidad de la internacionalización en sí misma (Sebastián, 2017). 
Tal como expresan Bordean y Borza (2013), desde un enfoque latinoamericano la internacionalización está enraizada con la globalización y este segundo fenómeno ha tendido a concentrar riqueza y conocimientos aunque la internacionalización no necesariamente debe seguir este camino. Didou (2017) expresa la importancia de analizar cómo América Latina está participando de la internacionalización, si es principalmente dirigida de norte a sur, qué colaboración sur-sur existe y si hay un intercambio desigual en el primer escenario.

\section{Metodología de indagación}

El documento persigue una estrategia de investigación cualitativa. Se realiza un abordaje descriptivo con la finalidad de conocer las características de los elementos analizados, lo que implica la recolección de la información sin establecer relaciones causales entre las dimensiones (Hernández, Fernández y Baptista, 2010). Si bien se trata de un estudio de caso en la medida en que se aborda el caso uruguayo, las dimensiones de análisis mencionadas pueden resultar útiles para analizar otros procesos de acuerdo al nivel político-administrativo de interés (municipio, provincia, país, región). El estudio de caso se caracteriza por intentar conocer su unicidad y está orientado a un enfoque de estudio intrínseco, esto es, comprender el caso en profundidad (Stake, 1995). Previamente se realizó una revisión de antecedentes bibliográficos, lo que pauta un diseño orientado al análisis documental (Corbetta, 2003).

\section{Aproximaciones a las dimensiones señaladas para el caso uruguayo}

En este segmento se presentan brevemente algunos elementos de análisis para el caso uruguayo de acuerdo a las dimensiones formuladas en el marco conceptual desarrollado en el capítulo 2.

\section{Diseño institucional}

La ET en Uruguay presenta novedades en el campo de las instituciones universitarias tras la aprobación de la LGE en 2008. La aprobación del Decreto-Ley N 15.661 (Parlamento, 1984) en el que se establecen disposiciones para los títulos profesionales que otorguen las universidades privadas desafió al monopolio de la Universidad de la República (UdelaR) en lo que respecta a la provisión del servicio educativo a nivel superior. El segundo desafío monopólico -esta vez en el ámbito público- se concreta con el impulso de esta legislación y posterior creación de la Universidad Tecnológica (UTEC) en 2012 (Bentancur y Clavijo, 2016).

Asimismo, el proceso de universitarización de la formación docente sufrió un duro revés en el año 2012 luego de que la Cámara de Representantes no aprobara el proyecto de creación del Instituto Universitario de Educación. Sin embargo, recientemente se ha incorporado a la agenda la consolidación de este proyecto. Asimismo la universitarización como proceso se encuentra en discusión en esferas tales como la educación policial y military atraviesa un proceso de reglamentación de la legislación vigente, así como también en el ámbito de las artes con la probable incorporación de la Escuela Multidisciplinaria de Arte Dramático a la UdelaR (Clavijo, 2016). Por su parte, la UdelaR avanzó en un proyecto democratizador a través de la denominada Segunda Reforma Universitaria durante el Rectorado de Rodrigo Arocena (2008-2014). Este proceso se caracterizó por el impulso del proceso de descentralización universitaria, la concepción del conocimiento como motor de desarrollo y la reivindicación de los pilares fundamentales de la institución: autonomía, cogobierno y democratización en el ingreso (Bentancur y Clavijo, 2016). 
Sin embargo, tales novedades pueden ser relativizadas en términos de diversificación institucional, esto es, la incidencia de las condiciones ambientales ante la posibilidad de cambios a nivel institucional (Landoni, 2017). Ciertamente, el centenario monopolio de la UdelaR en materia de ET puede incidir en la impronta de las políticas a ese nivel y, así, condicionar cambios institucionales.

Además de las instituciones per se es necesario atender a las alternativas de la organización institucional de las universidades en términos de estructura y de gobernanza. Cada universidad pública cuenta con su Consejo Directivo Central (CDC/CODICEN) cuya elección debe contemplar lo que establece el art. 203 de la Constitución de la República (Parlamento, 2004)ii.

En tanto, el Decreto 104 (Ministerio de Educación y Cultura, 2014) regula el ámbito privado aunque no avanza sustancialmente en aspectos de organización y estructura a excepción de las diferenciaciones entre una universidad y un instituto universitario pero sin incursionar en aspectos vinculados al organigrama de las universidades o de gobernanza institucional (art. $4^{\circ}$ ). Asimismo, de acuerdo al art. $6^{\circ}$ del mencionado Decreto se hace hincapié en la autorización para funcionar y el reconocimiento del nivel académico de dichas instituciones.

En materia de evaluación los artículos 113-119 de la LGE pautan la creación del INEEd y en 2011, a través de la Ley N 18.869, se modifica la integración de su Consejo Directivo por el cual el MEC agrega un segundo representante en el organismo en detrimento de un representante del CODICEN de la Administración Nacional de Educación Pública (ANEP). Por lo tanto dicho organismo de carácter público no estatal cuenta con un Consejo Directivo integrado por seis miembros: dos designados por el MEC (preside uno de ellos), dos electos por el CODICEN de la ANEP, un representante del Consejo Directivo Central (CDC) de la UDELAR y uno por la educación privada, inicial, primaria y media habilitada (Parlamento, 2011).

Un antecedente fundamental al que debemos remitir es la frustrada aprobación del proyecto de Ley que creaba la Agencia para la Promoción y Aseguramiento de la Calidad de la Educación Terciaria (APACET) orientada a la acreditación de la oferta educativa privada y terciaria externa a la institucionalidad de ANEP y UdelaR. Tal como señala Ares Pons (2009), el carácter monopólico de la UdelaR en ET hasta 1985 y la tardía emergencia de la oferta educativa privada postergaron este proceso de discusión. Por su parte, Landoni $(2010,2017)$ advierte las dificultades con respecto a la creación de la APACET, donde hay consensos entre los actores sobre la necesidad e importancia del asunto pero son divergentes los mecanismos de regulación. En 2009 el proyecto ingresa al Poder Legislativo pero finalmente se archiva (Parlamento, 2010).

Sin embargo cabe señalar que luego de un proceso que se retoma en 2018 a instancias del Grupo de Trabajo de Acreditación integrado por representantes del MEC, el Consejo de Formación en Educación (CFE) de la ANEP y de las universidades públicas y privadas se aprobó la creación del Instituto Nacional de Acreditación y Evaluación de la Educación Terciaria (INAEET) como persona jurídica de derecho público no estatal a través de la Ley $N^{\circ} 19.852$, la que entrará en vigencia a partir del $1^{\circ}$ de enero de 2021 (Parlamento, 2019).

En suma, las recientes transformaciones y las asignaturas pendientes marcan una complejidad primordial en términos del problema planteado. La atención de las estructuras y dinámicas institucionales es esencial. Existe un nivel macro limitado por 
disposiciones constitucionales en lo público y parcialmente por decreto en el ámbito privado. En su interior cada institución contempla sus propios organigramas. El ejercicio del cogobierno y la descentralización es característico de la UdelaR frente a estrategias de concurso en el ámbito de UTEC y CFE, mientras que las universidades privadas pautan sus propias reglas de juego. En este escenario fragmentado y disperso no resulta sencillo imaginar la evaluación desde un enfoque sistémico como consecuencia de la autonomía funcional de las instituciones involucradas, aunque sí se registran avances recientes en el ámbito de la acreditación.

\section{Territorialización}

Durante las dos últimas décadas las instituciones que conforman el sistema han presentado significativas acciones de descentralización. La justificación ha residido, en mayor o menor medida, en las desigualdades territoriales y en la necesidad de extenderse en todo el territorio (Bentancur y Clavijo, 2016).

Las instituciones pioneras en este proceso han sido el CETP-UTU y Formación Docente. Su rol de avanzada puede entenderse considerando su vínculo con la educación media, que ha generado una oferta en el interior del país que precede, por muchos años, a la descentralización universitaria (Lizbona y Rumeau, 2013). CETP-UTU crea cinco regiones denominadas Campus Regionales de Educación Tecnológica que implican 44 centros educativos, además de Montevideo y Canelones. A su vez, el CFE crea cinco regiones además de la Región Centro Sur, lo que implica 24 centros educativos entre los IFD y los CeRP (Cánepa y López, 2014).

La UdelaR planificó su descentralización desde un enfoque de regionalización territorial. Es pertinente considerar al año 2007 como hito que marca la etapa de descentralización en la que la UdelaR se encuentra actualmente. Como antecedentes fundamentales están los Planes Estratégicos de Desarrollo de la Universidad de la República (Pledur) elaborados en los años 2000 y 2005, donde se expresa la voluntad explícita de impulsar esta política (Contera, 2008). En 2007, el CDC de la UdelaR aprobó un conjunto de resoluciones que implicaron un cambio de rumbo en el programa de desarrollo de la UdelaR en el interior del país: la creación de instituciones autónomas, cogobernadas y distribuidas en el territorio nacional, la promoción de la radicación de docentes y el afianzamiento o creación de grupos de investigación en el interior, la implementación de programas de carácter regional así como programas de formación específicos de alcance nacional (Figueroa, 2016). La UdelaR creó instituciones en tres regiones: Centro Universitario Regional Litoral Norte, Centro Universitario Regional Este y Centro Universitario Regional Noreste, lo que implica un total de 11 instituciones educativas en el norte y este del territorio nacional.

Por su parte la UTEC concibe la necesidad de llevar a cabo un proceso de regionalización desde su fundación. La Ley de creación señala explícitamente que "la sede central de la UTEC estará ubicada en el interior del país"iii. Su organización territorial establece Institutos Tecnológicos Regionales (ITR). En 2014 la UTEC lanzó sus dos primeras ofertas académicas en el interior del país: Licenciatura en Leche y Productos Lácteos y Licenciatura en Análisis Alimentario. En ambos casos se trató de formación a nivel de grado universitario orientada a dar continuidad a propuestas de enseñanza terciaria no universitaria ofrecidas por otras instituciones del Sistema Nacional de Educación Terciaria Pública (SNETP) (Cánepa y López, 2014; Universidad Tecnológica, 2016). 
La Coordinadora del Sistema Nacional de Educación Terciaria Pública (CSNETP) realizó un esfuerzo por sistematizar y discutir la regionalización de las distintas instituciones que pertenecen al sistema. Uno de los objetivos explícitos de este esfuerzo fue el de compartir recursos físicos y humanos de todos los integrantes del SNETP (Cánepa y López, 2014).

En cuanto a las universidades privadas, algunas de ellas se han embarcado en el objetivo de instalar sedes en el interior del país. Tal es el caso de la Universidad Católica que desde 1989 cuenta con una sede en la ciudad de Maldonado y en 2003 se instaló en la ciudad de Salto. En el año 2017 además de Montevideo, los departamentos con presencia de universidades privadas son Salto, Maldonado y Colonia (Ministerio de Educación y Cultura, 2017).

En resumen, las instituciones públicas de ET han perseguido procesos de descentralización de carácter regional con el objetivo de reducir las inequidades en el acceso al sector educativo. La presencia de las universidades privadas en el interior complementa y diversifica la oferta educativa pública, aunque con criterios propios del sector privado.

\section{Aspectos sociodemográficos}

Conocer las características sociodemográficas de los estudiantes involucrados en los sistemas educativos es útil a los efectos de evaluar la equidad en el sistema (Formichella, 2011). Existe un consenso en considerar aspectos económicos y sociales básicos tales como nivel socioeconómico de la familia, sexo y edad de los estudiantes, por un lado para evaluar la equidad en el sistema y por otro para caracterizar a la población y orientar las decisiones en este sentido.

El relevamiento de esta información depende de las disposiciones de cada institución educativa, que llevan a cabo diversas estrategias de recolección. No todas las instituciones realizan un formulario de ingreso y, en caso de que se realicen, las mismas priorizan ciertos contenidos. En consecuencia, más allá de los esfuerzos que realiza cada institución es imprescindible considerar instancias de coordinación para la recolección de información de esta naturaleza, lo que colaboraría en su armonización. Este obstáculo se presenta también en la dimensión que se desarrolla a continuación.

\section{Aspectos del proceso educativo}

Conviene analizar el proceso educativo, los recursos y los resultados en base a las categorías y dimensiones del sistema de indicadores de Estévez y Pérez (2007). Ello implica constatar la situación inicial de los estudiantes y dar cuenta, a su vez, del proceso administrativo, organizacional, docente y pedagógico del programa.

Tal como se señaló anteriormente las instituciones apelan a diversas estrategias de generación y recolección de datos. En el caso de la UdelaR, docentes se encuentran trabajando en la generación de un sistema de indicadores para la enseñanza de grado, coordinado a nivel central a través de la Dirección General de Planeamiento (DGPLAN). El objetivo es crear un Sistema de Indicadores para la Evaluación Universitaria (SIEU) que permita evaluar las funciones de enseñanza de la UdelaR. Asimismo la Comisión Sectorial de Enseñanza (CSE) y la Comisión Coordinadora del Interior (CCI) han realizado aportes en la materia. Sin embargo, la evaluación en aprendizajes en ET resulta una asignatura pendiente. 
No obstante, los esfuerzos por armonizar información son escasos. En la UdelaR se establece un formulario de perfil de ingreso y se aplican censos universitarios. En CETPUTU no se implementa un formulario al ingreso de la institución. El CFE ha realizado censos y algunos trabajos, aunque no son periódicos. La UTEC realizó un censo a sus estudiantes y realiza memorias anuales donde reporta este tipo de datos. Por su parte, el Ministerio de Educación y Cultura (MEC) publica anualmente estadísticas agregadas sobre matrícula, ingreso y egreso, entre otros, tanto de las instituciones públicas como privadas.

\section{Aspectos curriculares}

Dentro de la evaluación del sistema de ET se identificó como fundamental la posibilidad de evaluar los programas ofrecidos por el sistema de ET en términos curriculares. Se constatan los siguientes aspectos: títulos otorgados, carga horaria, sistema de créditos, requisitos de ingreso, perfil de egreso, modalidades educativas, orientaciones curriculares, dispositivos de evaluación, estructura de los planes de estudios, validación de conocimiento y continuidad educativa.

Una experiencia concreta en la materia es el trabajo realizado por una comisión técnica con participantes de todas las instituciones públicas en la órbita del CSNETP en conjunto con el MEC. Estos actores institucionales se abocaron a la tarea de analizar la navegabilidad entre las instituciones de ET y los programas conjuntos ofrecidos, además de generar insumos con el objetivo de dilucidar la navegabilidad del sistema (Coordinación del Sistema Nacional de Educación Terciaria Pública, 2014).

Se consideró innovadora la sistematización de esta información desde una perspectiva sistémica y se generó un marco normativo que define y amplía la cooperación desde la identidad de cada institución. El objetivo fue generar mecanismos que habiliten la movilidad estudiantil y la construcción de un Sistema Nacional de Educación Terciaria colocando el foco en el estudiante. Ello requiere que las instituciones parte del sistema se conozcan y se reconozcan (Coordinación del Sistema Nacional de Educación Terciaria Pública, 2016).

En el ámbito privado es el Consejo Consultivo de Educación Terciaria Privada el que ejerce competencia en la autorización para que las universidades e institutos universitarios privados funcionen y les sea reconocido su nivel académico, de conformidad con el art. $6^{\circ}$ del Decreto 104 (Ministerio de Educación y Cultura, 2014).

En suma sería deseable la replicación de estas experiencias en un contexto de articulación público/privada. Ello implicaría avanzar en la consolidación de un sistema de ET para lograr la armonización de información y la homogeneización de criterios de titulación, revalidación de materias y creditización, entre otros.

\section{Financiamiento}

El sistema de ET uruguayo se caracteriza por ser de provisión mixta, con componentes públicos y privados, lo que tiene obvias implicaciones para las diversas modalidades de financiamiento. A pesar de la preponderancia de lo público es necesario poder distinguir entre las fuentes de financiamiento para las diferentes instituciones y su composición, a modo de poder evaluar esta dimensión a nivel de sistema. 
La fuente de financiamiento acarrea grandes implicancias económicas y políticas. El aspecto económico debería poder garantizar sostenibilidad en el tiempo, una característica clave del proyecto educativo. A su vez el gasto público en educación conlleva un matiz de equidad. Según un estudio de la CEPAL para América Latina, el gasto público total en ET es de carácter regresivo, beneficiando mayoritariamente a la población más pudiente (Téllez y Rodríguez, 2003).

La principal fuente de financiamiento de las instituciones de ET públicas se da a través de asignaciones directas del presupuesto nacional, lo que refuerza la importancia de la misma a nivel país tanto desde un enfoque de derechos como en un sentido de rentabilidad general de la ET. Este mecanismo funciona bajo un modelo histórico-negociado por el cual se negocian incrementos presupuestales con base en años anteriores. Una de las mayores críticas a este proceso político es la ausencia de incentivos para mejorar la eficiencia del dinero o la calidad del servicio prestado (García Guadilla, 2007).

Por una parte, en el caso de la oferta privada es necesario destacar que se financia mayormente a través de su matrícula (Oddone y Perera, 2004). Este mecanismo de financiamiento en Uruguay se encuentra restringido para todas las instituciones públicas (art. 71 de la Constitución de la República). Asimismo cabe destacar que el art. 69 exonera a las instituciones de ET privadas de impuestos nacionales y departamentales como mecanismo de subvención por el servicio brindado (Parlamento, 2004).

Por otra parte se relevan para ambos tipos de institución otras fuentes de financiamiento (fondos concursables, convenios sectoriales, legajos y donaciones, financiamiento indirecto a través de exenciones tributarias, etc.) de menor peso.

En definitiva, el financiamiento de la ET en Uruguay adopta rasgos particulares en un sistema de naturaleza mixta de provisión del servicio, con diversidad de fuentes y esquemas compartidos. Sería de rigor analizar los diversos modos de financiamiento y la composición del ingreso con base en tres criterios: su sostenibilidad, los incentivos que la fuente genera en pos de la eficiencia y la equidad económica del financiamiento.

\section{Internacionalización}

En el concierto latinoamericano se ha desarrollado un conjunto de experiencias regionales, entre las que pueden citarse las siguientes: el Programa de Intercambio y Movilidad Académica impulsado por la Organización de Estados Iberoamericanos para la Educación, la Ciencia y la Cultura (OEI), El Programa Escala Estudiantil de la Asociación de Universidades del Grupo Montevideo (AUGM), el Programa Regional MERCOSUR (MARCA), que involucra carreras de grado acreditadas en el ARCU-SUR y en el que participan universidades uruguayas así como de Argentina, Brasil, Bolivia, Paraguay, Chile y Venezuela (Cánovas et al., 2017).

En tanto, las diferentes experiencias institucionales en Uruguay sugieren estrategias de internacionalización heterogéneas de inserción regional e internacional. Esto respondería a la fragmentación y diversificación institucionales antes señaladas, lo que dificulta la caracterización de la internacionalización de la ET en nuestro país. En este sentido se considera pertinente que las instituciones categoricen las estrategias institucionales existentes para lograr cooperación y movilidad de estudiantes y académicos. 


\section{Comentarios finales: conocimiento, reconocimiento y consenso entre actores}

En estas líneas se ha realizado un esfuerzo por identificar nudos que anteceden a la formulación de dimensiones, categorías e indicadores robustos para la concreción de una evaluación sistémica. Las dimensiones problematizadas no son exhaustivas.

Tales políticas cobran mayor relevancia en un contexto de diversificación institucional de la ET cuyo alcance refiere a instituciones, propuestas y actores educativos. A su vez, el impulso democratizador que ha experimentado la ET en Uruguay, traducido en el aumento de la matrícula y la expansión de la oferta en todo el país, requiere de una evaluación que permita dar cuenta de la calidad educativa.

En virtud de las dimensiones señaladas pueden identificarse ciertos desafíos en el campo de la ET en Uruguay, a saber:

- Atender a los diseños institucionales, tanto como objeto de evaluación como también en la conformación de instancias de participación y diálogo entre actores, las que deben contemplar la normativa consagrada constitucionalmente.

- Fortalecer el proceso de territorialización tanto en el sector público como privado, lo que contribuye a la formación de capital humano en todo el territorio nacional. Si bien existen antecedentes históricos en la materia, las reformas implementadas durante la última década han derivado en nuevos desafíos en el campo de la ET, que interactúan con algunas de las dimensiones aquí descritas.

- Colocar en agenda el estudio de trayectorias educativas atendiendo a las características sociodemográficas de quienes se incorporan a la ET es indispensable, por lo que sería deseable la implementación de instrumentos comunes de relevamiento de este tipo de información.

- Propender a la colaboración interinstitucional hacia la evaluación y el monitoreo de los procesos de enseñanza-aprendizaje. Para ello es necesario avanzar en la construcción de indicadores que contemplen las particularidades de las instituciones del sistema considerando aspectos tales como el tipo de provisión del servicio, su estructura académica, los ingresos, matrículas, actividades curriculares y egresos de los estudiantes, así como también la calificación del elenco docente, entre otros posibles ejes.

- En el ámbito curricular, colocar el foco en el estudiante. En este sentido, atender a la navegabilidad entre instituciones de ET así como también a la complementación de la oferta curricular además de establecer mecanismos comunes de reválidas entre instituciones del sistema (por ejemplo, desarrollando un sistema de creditización común) bajo el principio de equivalencia razonable.

- Avanzar en el monitoreo del financiamiento de la ET atendiendo a principios de sostenibilidad, eficiencia y equidad económica. 
- Retomar el debate acerca de los procesos de internacionalización de la ET. Por un lado, estimular y jerarquizar en la agenda las instancias de cooperación y movilidad estudiantil y académica con instituciones educativas terciarias a nivel internacional. Por otro, reflexionar acerca de las implicancias de los enfoques mercantilizadores de la internacionalización de la ET dado que en el art. 71 de la Carta Magna se establece su utilidad social (Parlamento, 2004).

En suma este abordaje pretende ser un insumo para una discusión de la temática que cuente con la participación de la comunidad educativa, la sociedad civil, los partidos políticos y el gobierno nacional entre otros actores de relevancia. Se considera de particular interés una evaluación integral del sistema de ET a los efectos de poder optimizar recursos y prácticas institucionales. Para ello es necesario que los actores del sistema logren conocerse y reconocerse hacia la discusión y concreción del objetivo planteado.

\section{Bibliografía}

Altbach, P. y Knight, J. (2007). The internationalization of higher education: motivation and realities. Journal of Studies in International Education, 290-305.

Archer, L. (2003). Higher Education and Social Class. Issues of exclusion and inclusion. London and New York: Routledge Falmer.

Ares Pons, J. (2009). La regulación de la enseñanza terciaria en el Uruguay. Historia y situación actual. Montevideo: Documento de trabajo presentado en el seminario "La evaluación y acreditación en instituciones de educación superior. Autoevaluación y evaluación externa, desafíos e instrumentos". Disponible en: http://entre-dos.org/sites/default/ files/La\%20evaluaci\%C3\%B3n\%20y\%20Acreditaci\%C3\%B3n\%20en\%20Instituciones\%20 de\%20Educaci\%C3\%B3n\%20Superior...Ares\%20Pons.pdf

Bentancur, N. (2012). Aporte para una topografía de las políticas educativas en Uruguay: Instituciones, ideas y actores. Revista Uruguaya de Ciencia Política (21)(1), 65-92. Disponible en: www.redalyc.org/articulo.oa?id=297325499004

Bentancur, N. y Clavijo, E. (2016). La educación superior durante el decenio frenteamplista: análisis de un caso de innovación en las políticas. Ponencia presentada a las XV Jornadas de Investigación Científica de la Facultad de Ciencias Sociales, UdelaR. Montevideo, 14-16 de setiembre de 2016. Disponible en: http://jornadas.cienciassociales.edu.uy/wp-content/ uploads/2016/10/Eje_El-decenio-progresista.-Las-pol\%C3\%ADticas-p\%C3\%BAblicas-_ Bentancur-Clavijo.pdf

Bordean, O. N. y Borza, A. (2013). Internationalization of Higher Education Institutions: The Case of Romania. Procedia - Social and Behavioral Sciences, 98-103.

Brunner, J. y Villalobos, C. (2014). Políticas de educación superior en Iberoamérica, 20092013. Centro de Políticas Comparadas de Educación, Universidad Diego Portales, Cátedra Unesco de Políticas Comparadas de Educación Superior, Santiago de Chile. Disponible en: https://searchworks.stanford.edu/view/10770206

Cabella, W., Fernández, M. y Prieto, V. (2015). Las transformaciones de los hogares uruguayos vistas a través de los censos de 1996 y 2011. Atlas sociodemográfico y de la desigualdad del Uruguay, 6, Instituto Nacional de Estadística, Uruguay. 
Calero, J. (2006). La equidad en educación. Informe analítico del sistema educativo español. Centro de Investigación y Documentación Educativa (CIDE). Disponible en: redined.mecd. gob.es/xmlui/handle/11162/61929

Cánepa, G. y López, A. (Coords.) (2014). Aportes para la construcción de un sistema de educación terciaria pública. Sistema Nacional de Educación Terciaria Pública, Montevideo. Disponible en: http://www.cfe.edu.uy/images/stories/pdfs/publicaciones/2014/aportes para_construccion_snep.pdf

Cánovas, L., Maure, E. y P. de Borbón, L. (2017). Internacionalización de la educación superior: la movilidad estudiantil como principal estrategia. Políticas Educativas, 11(1), 153-162.

Centro Interuniversitario de Desarrollo (2008). Diseño curricular basado en competencias y aseguramiento de la calidad en la educación superior. Santiago de Chile. Disponible en: https://cinda.cl/publicacion_archivos/diseno-curricular-basado-en-competencias-yaseguramiento-de-la-calidad-en-la-educacion-superior

Centro Interuniversitario de Desarrollo (2014). Evaluación del aprendizaje en innovaciones curriculares de la Educación Superior. Santiago de Chile. Disponible en: https://cinda.cl/ publicacion_archivos/evaluacion-del-aprendizaje-en-innovaciones-curriculares-de-laeducacion-superior

Chowdry, H., Crawford, C., Dearden, L., Goodman, A. y Vignoles, A. F. (2008). Widening participation in higher education: analysis using linked administrative data. IFS Working Paper W10/04. Economic and Social Research Council.

Clavijo, E. (2016). El mosaico de la universitarización en el Sistema Nacional de Educación Terciaria Pública. Trabajo presentado en las Jornadas de Investigación de la Facultad de Ciencias Sociales de la Universidad de la República. Montevideo, 12, 13 y 14 de setiembre de 2016. Disponible en: http://jornadas.cienciassociales.edu.uy/wp-content/ uploads/2016/10/Eje_-Educaci\%C3\%B3n-I-_-EmilianoClavijo.pdf

Contera, C. (2008). La educación superior en Uruguay. Avaliação, 13(2), 533-554.

Coordinación del Sistema Nacional de Educación Terciaria Pública (2014). Abrir las puertas del sistema (Acta N9). Documento de trabajo. Montevideo: Ministerio de Educación y Cultura.

Coordinación del Sistema Nacional de Educación Terciaria Pública (2016). Acta N8. Montevideo: Ministerio de Educación y Cultura.

Corbetta, P. (2003). Metodología y técnicas de investigación social. Madrid: McGraw-Hill.

Dente, B. y Subirats, J. (2014). Decisiones públicas. Análisis y estudio de los procesos de decisión en políticas públicas. Barcelona: Ariel.

Díaz Barriga, Á. (2005). Evaluación curricular y evaluación de programas con fines de acreditación. Cercanías y desencuentros. Congreso Nacional de Investigación Educativa. Sonora. UNAM, México. Disponible en: www.angeldiazbarriga.com/ponencias/ conferencia_cnie2005.pdf 
Didou Aupetit, S. (2017). La internacionalización de la educación superior en América Latina: transitar de lo exógeno a lo endógeno. México: Unión de Universidades de América Latina y el Caribe.

Di Gropello, E. (2004). La descentralización de la educación y las relaciones de rendición de cuentas en los países latinoamericanos. PREAL. Disponible en: https://www.oei.es/historico/ reformaseducativas/descentralizacion_educacion_rendicion_cuentasAL_digropello.pdf

Esquetini, C. y Rodríguez, E. (2012). Estructura y titulaciones de Educación Superior en Ecuador. OEl. Disponible en: https://www.oei.es/historico/homologaciones/ecuador.pdf

Estévez, J. F. y Pérez, M. J. (2007). Sistema de indicadores para el diagnóstico y seguimiento de la educación superior en México. ANUIET: México.

Figueroa, V. (2016). Construcción de la oferta académica universitaria en el interior del país en el marco de la política de la descentralización de la Universidad de la República. En G. Carreño (Coord.). Logros de la UdelaR en el Interior del país 2005-2015. Montevideo: Comisión Coordinadora del Interior. Disponible en: www.cci.edu.uy/sites/default/files/ Logros_2016con_tapa.pdf

Formichella, M. (2011). Análisis del concepto de equidad educativa a la luz del enfoque de las capacidades de Amartya Sen. Revista Educación, 35, 15-34.

García Guadilla, C. (2007). Financiamiento de la Educación Superior en América Latina. Sociologias, 17(1), 50-101. Disponible en: http://www.scielo.br/scielo.php?pid=S1517$\underline{45222007000100004 \& \text { script }=\text { sci_abstract\&tlng=es }}$

Hall, P. (1993). Policy Paradigms, Social Learning, and the State: The Case of Economic Policymaking in Britain. Comparative Politics, 25(3), 275-296. Disponible en: https://www. jstor.org/stable/422246

Hernández, R., Fernández, C. y Baptista, P. (2010). Metodología de la Investigación. México: McGraw-Hill.

Knight, J. (2015). International Universities: Misunderstandings and Emerging Models? Journal of Studies in International Education, 19(2), 107-121.

Landoni-Couture, P. (2010). El debate sobre la creación de una Agencia de Acreditación en Uruguay. Influencias externas y tensiones internas. Revista Argentina de Educación Superior, 2, 96-110. Disponible en: https://dialnet.unirioja.es/servlet/articulo?codigo=6488174

Landoni-Couture, P. (2017). Procesos de diversificación institucional en la educación superior uruguaya: avances y frustraciones. Ponencia presentada al $9^{\circ}$ Congreso Latinoamericano de Ciencia Política, organizado por ALACIP. Montevideo.

Disponible en: http://www.congresoalacip2017.org/arquivo/downloadpublic2?q=YToy OntzOjY6InBhcmFtcyl7czozNToiYToxOntzOjEwOiJJRF9BUIFVSVZPIjtzOjQ6ljIwMzAiO30i O3M6MToiaCl7czozMjoiNWE0ZjdhZDRjODYxZTA3ZmQ1 MjA2MjYOYThhYWRiNDQiO30 $\underline{\%}$ D

Lindblom, C. (1992). La ciencia de salir del paso. En: Aguilar Villanueva, L. (Ed.). La hechura de las políticas, 201-225. México: Miguel Ángel Porrúa Grupo Editorial. 
Lizbona, A. y Rumeau, D. (2013). La educación universitaria en el Uruguay: continuidades, cambios y desafíos (1960-2012). Montevideo: INEEd. Disponible en: https://www.ineed. edu.uy/images/pdf/la-educacion-universitaria-uruguay.pdf

March, J. G. y Olsen, J. P. (1989). Rediscovering institutions: The organizational basis of politics. New York: Free Press.

Marquina, M. (2004). Panorama de las titulaciones en el sistema de Educación Superior Argentino: Aportes para un estudio comparado. Buenos Aires: Comisión Nacional de Evaluación y Acreditación Universitaria. Disponible en: https://www.yumpu.com/es/ document/view/14894596/panorama-de-las-titulaciones-en-el-sistema-coneau

Marsh, D. y Rhodes, R. A. W. (1992). Policy Networks in British Government. Oxford: Clarendon Press.

Ministerio de Educación y Cultura (2014). Decreto 104. Reglamentación del Decreto Ley 15.661 relativo al Sistema de Enseñanza Terciaria Privada. Montevideo: IMPO. Disponible en: https://www.impo.com.uy/bases/decretos/104-2014/19

Ministerio de Educación y Cultura (2017). Universidades y carreras privadas 2017. Disponible [actualizado] en: https://www.gub.uy/ministerio-educacion-cultura/sites/ ministerio-educacion-cultura/files/2020-06/Universidades\%20y\%20Carreras\%20 JUNIO\%202020.pdf

Oddone, G. y Perera, M. (2004). Educación Superior en Uruguay: descripción y financiamiento. Montevideo: CINVE. Disponible en: www.cinve.org.uy/wp-content/ uploads/2013/01/educacion-superior-en-uruguay.pdf

Paredes, M. y Varela, C., (2005). Aproximación sociodemográfica al comportamiento reproductivo y familiar en Uruguay, Documento de trabajo $N^{\circ}$ 67. Montevideo: Unidad Multidisciplinaria, Facultad de Ciencias Sociales, Universidad de la República. Disponible en: $\quad$ https://www.colibri.udelar.edu.uy/jspui/bitstream/20.500.12008/4631/1/DT\%20 MULTI\%2067.pdf

Parlamento (1984). Ley $N^{\circ}$ 15.661. Se establecen disposiciones para los títulos profesionales que otorguen las Universidades Privadas. Montevideo: Parlamento. Disponible en: https:// parlamento.gub.uy/documentosyleyes/leyes?Ly Nro=15661\&Ly fechaDePromulgacion\% 5Bmin\%5D\%5Bdate\%5D=25-02-1983\&Ly fechaDePromulgacion\%5Bmax\%5D\%5Bdate\% 5D=26-02-2020\&Ltemas=\&tipoBusqueda=T\&Searchtext $=$

Parlamento (2004). Constitución de la República Vigente. Montevideo: Parlamento. Disponible en: https://parlamento.gub.uy/documentosyleyes/documentos/11/ HTML?width=800\&height $=600 \&$ hl=en US1\&iframe $=$ true\&rel $=0$

Parlamento (2008). Ley $N^{\circ}$ 18.437. Ley General de Educación. Montevideo: Parlamento. Disponible en: https://parlamento.gub.uy/documentosyleyes/leyes/ley/18437?width=80 0\&height=600\&hl=en_US1\&iframe=true\&rel=nofollow

Parlamento (2010). Diario de Sesiones de la Cámara de Representantes $N^{\circ} 3630$. Montevideo: Parlamento. Disponible en: https://parlamento.gub.uy/documentosyleyes/ documentos/diario-de-sesion/representantes/3237/IMG/0?width=800\&height=600\&hl= en US1\&iframe=true\&rel=nofollow 
Parlamento (2011). Ley $N^{\circ}$ 18.869. Comisión Directiva del Instituto Nacional de Evaluación Educativa. Modificación de su integración. Montevideo: Parlamento. Disponible en: https:// parlamento.gub.uy/documentosyleyes/leyes/ley/18869

Parlamento (2012). Ley $N^{\circ}$ 19.043. Universidad Tecnológica. Montevideo: Parlamento. Disponible en: https://parlamento.gub.uy/documentosyleyes/leyes/ley/19043?width=80 0\&height=600\&hl=en_US1\&iframe=true\&rel=nofollow

Parlamento(2019).Ley N¹9.852. Instituto NacionaldeAcreditacióny Evaluación dela Educación Terciaria (INAEET). Creación. Disponible en: https://parlamento.gub.uy/documentosyleyes/ leyes/ley/19852?width=800\&height $=600 \&$ hl=en_US1\&iframe=true\&rel=nofollow

Pierson, P. y Skocpol, T. (2008). El institucionalismo histórico en la ciencia política contemporánea. Revista Uruguaya de Ciencia Política, 17, 7-38. Disponible en: http://www. scielo.edu.uy/pdf/rucp/v17n1/v17n1a01.pdf

Rama, C. (2015). Nuevas formas de regionalización de la educación superior en América Latina: Las Universidades Red y los Cluster Universitarios. Revista Gestão Universitária na América Latina, 8(2). Disponible en: https://www.redalyc.org/articulo.oa?id=319338455015

Rangel, A. (2011). La descentralización de la Educación Superior. Anuies Revista de la Educación Superior. Disponible en: http://publicaciones.anuies.mx/pdfs/revista/Revista19. S1A4ES.pdf

Ravela, P. (2016). Para comprender las evaluaciones educativas. Fichas didácticas. Programa de Promoción de la Reforma Educativa en América Latina (PREAL). Disponible en: https://www.thedialogue.org/wp-content/uploads/2016/07/2006-Introduccion-Paracompreender-las-evaluaciones-educativas-Fichas-didacticas-Ravela.pdf

Salazar, C. y Chiang, M. (2007). Competencias y educación superior. Un estudio empírico. Horizontes Educacionales (12), 23-35.

Schmidt, V. (2008). Discursive Institutionalism: The Explanatory Power of Ideas and Discourse. Annual Review of Political Science, 11, 303-326.

Sebastián, J. (2017). Algunos dilemas en torno a la internacionalización de la Educación Superior. Internacionalización de la Educación Superior, 21(21), 119-149.

Shavit, Y., Arum, R. y Gamoran, A. (eds.) (2007). Stratification in Higher Education. A Comparative Study. Stanford: Stanford University Press.

Smyth, E. (2003). Gender differentiation and early labour market integration across Europe. En Muller, I. K., School-to-work Transitions in Europe: Analyses of the EULFS 2000 Ad Hoc Module. Mannheim.

Stake, R. E. (1995). Investigación con estudio de casos. Madrid: Morata.

Téllez, J. y Rodríguez, F. (2003). Crédito educativo: experiencias internacionales y desafíos futuros en América Latina. Bogotá: APICE. Disponible en: https://docplayer.es/3129189Credito-educativo-experiencias-internacionales-y-desafios-futuros-en-america-latina. $\underline{\mathrm{html}}$ 
Trylesinski, F. (2006). Gasto Público y Privado en Salud Distribución Territorial. Montevideo: Convenio Institucional entre la Federación Médica del Interior y la Universidad de la República. Disponible en: https://c6c493a3-18e5-4bd1-92b4-dc602742389c.filesusr.com/ ugd/222cdb_63d85a0bb93946ed98b13c5953dd89aa.pdf

Unidad de Sistemas de la Información de la Enseñanza. (2014). Documento de Trabajo N²: Sistema de indicadores de la enseñanza. Montevideo: Comisión Sectorial de Enseñanza UDELAR.

Universidad Tecnológica (2016). Memoria Anual 2015. Montevideo: Imprimex. Disponible en: https://utec.edu.uy/es/memoria-anual-2015/

\section{(c)) BY}

Este artículo está sujeto a una licencia internacional Creative Commons Attribution 4.0.

*.** ${ }^{* * *}$ Universidad de la República, Uruguay

${ }^{1}$ Magíster (Cand.) en Ciencia Política, Licenciado en Ciencia Política y Licenciado en Sociología, Docente ayudante, Instituto de Ciencia Política, Facultad de Ciencias Sociales, Universidad de la República (Uruguay).

${ }^{2}$ Magíster en Sociología y Licenciada en Sociología, Universidad de la República (Uruguay).

${ }^{3}$ Magíster (Cand.) en Economía, Universidad de la República (Uruguay). Licenciada en Economía Internacional y Desarrollo, Universidad de Bayreuth (Alemania). Asistente de investigación, Instituto de Economía, Facultad de Ciencias Económicas y de Administración, Universidad de la República (Uruguay).

\section{Notas}

Aprobación final del artículo, editora responsable Mag. Verónica Zorrilla de San Martín

Contribución de autoría: Los autores han realizado el artículo por partes iguales.

'Entrevista disponible en: http://radiouruguay.uy/la-UdelaR-redoblara-su-apuesta-por-laspropuestas-academicas-en-el-interior-dijo-arim/

"La UTEC presenta un CDC de carácter provisorio (Parlamento, 2012). El CFE presenta una figura análoga a un CDC aunque no se trate de un ente autónomo en sí mismo.

iii'Art. $1^{\circ}$, Ley Nº19.043 (Parlamento, 2012). Actualmente, su sede central se ubica en la ciudad de Montevideo. 